\title{
Difference inequality for stability of impulsive difference equations with distributed delays
}

\author{
Dingshi $\mathrm{Li}^{1 *}$, Shujun Long ${ }^{2}$ and Xiaohu Wang ${ }^{\text {* }^{*}}$
}

* Correspondence:

lidingshi2006@163.com;

xiaohuwang1111@163.com

${ }^{1}$ Yangtze Center of Mathematics,

Sichuan University, Chengdu

610064, P. R. China

Full list of author information is

available at the end of the article

\begin{abstract}
In this paper, we consider a class of impulsive difference equations with distributed delays. By establishing an impulsive delay difference inequality and using the properties of " $\rho$-cone" and eigenspace of the spectral radius of non-negative matrices, some new sufficient conditions for global exponential stability of the impulsive difference equations with distributed delays are obtained. An example is given to demonstrate the effectiveness of the theory.
\end{abstract}

Keywords: Difference equations, Impulsive, Distributed delays, Difference inequality, Global exponential stability

\section{Introduction}

Difference equations usually appear in the investigation of systems with discrete time or in the numerical solution of systems with continuous time [1]. In recent years, the stability investigation of difference equations has been interesting to many investigators, and various advanced results on this problem have been reported [2,3]. However, almost all available results have been focused on systems with discrete delays. In reality, difference systems with distributed delays become important because it is essential to formulate the discrete-time analogue of the continuous-time system with distributed delays when one wants to simulate or compute the continuous-time one after obtaining its dynamical characteristics. Fortunately, such an issue has been addressed in [4-7].

However, besides the delay effect, an impulsive effect likewise exists in a wide variety of evolutionary processes in which states are changed abruptly at certain moments of time, involving such fields as medicine, biology, economics, mechanics, electronics, and telecommunications. Recently, the asymptotic behaviors of impulsive difference equations have attracted considerable attention. Many interesting results on impulsive effect have been obtained [8-11].

It is well known that distributed delay differential equations with impulses or without impulses have been considered by many authors (see, for instance [12-14]). But, to the best of our knowledge, there is no concerning on the stability of impulsive difference equations with distributed delays in literature. Motivated by the above discussion, we here make a first attempt to arrive at results on the global exponential stability of impulsive difference equations with distributed delays.

\section{SpringerOpen ${ }^{\circ}$}

(C) $2011 \mathrm{Li}$ et al; licensee Springer. This is an Open Access article distributed under the terms of the Creative Commons Attribution License (http://creativecommons.org/licenses/by/2.0), which permits unrestricted use, distribution, and reproduction in any medium, provided the original work is properly cited. 


\section{Model description and preliminaries}

Let $R^{n}\left(R_{+}^{n}\right)$ be the space of $n$-dimensional (non-negative) real column vectors and $R^{m \times n}\left(R_{+}^{m \times n}\right)$ denotes the set of $m \times n$ (non-negative) real matrices. Usually, $E$ denotes an $n \times n$ unit matrix. For $A, B \in R^{m \times n}$ or $A, B \in R^{n}$, the notation $A \geq B(A>B)$ means that each pair of corresponding elements of A and B satisfies the inequality " $\geq$ (>)". Especially, $A \in R^{m \times n}$ is called a nonnegative matrix if $A \geq 0$, and $z \in R^{n}$ is called a positive vector if $z>0$. $Z$ denotes the integer set, $Z_{\infty}=\{j \in Z \mid-\infty<j \leq 0\}$ and $Z_{\infty}^{+}=\{j \in Z \mid 0 \leq j<\infty\} . C$ denotes the set of all bounded functions $\phi(j) \in R^{n}, j \in Z_{\infty}$.

For $x \in R^{n}, A \in R^{n \times n}, \phi \in C$, we define

$$
\begin{aligned}
& {[x]^{+}=\left(\left|x_{1}\right|, \ldots,\left|x_{n}\right|\right)^{T}, \quad[A]^{+}=\left(\left|a_{i j}\right|\right)_{n \times n,}} \\
& {[\varphi(m)]_{\infty}=\left(\left[\varphi_{1}(m)\right]_{\infty}, \ldots,\left[\varphi_{n}(m)\right]_{\infty}\right)^{T}, \quad[\varphi(m)]_{\infty}^{+}=\left[[\varphi(m)]^{+}\right]_{\infty}}
\end{aligned}
$$

where $\left[\varphi_{i}(m)\right]_{\infty}=\sup _{s \in Z_{\infty}}\left\{\varphi_{i}(m+s)\right\}$, and introduce the corresponding norm for them as follows:

$$
\|x\|=\max _{1 \leq i \leq n}\left\{\left|x_{i}\right|\right\}, \quad\|A\|=\max _{1 \leq i \leq n} \sum_{j=1}^{n}\left|a_{i j}\right|, \quad\|\varphi\|=\max _{1 \leq i \leq n}\left\{\left[\varphi_{i}(m)\right]_{\infty}^{+}\right\} .
$$

In this paper, we mainly consider the following impulsive difference equations with distributed delays

$$
\left\{\begin{array}{l}
x_{i}(m+1)=a_{i} x_{i}(m)+\sum_{j=1}^{n} b_{i j} f_{j}\left(x_{j}(m)\right)+\sum_{j=1}^{n} c_{i j} \sum_{k=1}^{\infty} \mu_{i j}(k) g_{j}\left(x_{j}(m-k)\right), \quad m \in Z_{\infty}^{+}, \quad m \neq m_{k \prime} \\
x_{i}(m+1)=H_{i m}\left(x_{1}(m), \ldots, x_{m}(m)\right), \quad m=m_{k} \\
x_{i}(m)=\varphi_{i}(m), \quad m \in Z_{\infty}
\end{array}\right.
$$

where $0<i \leq n$ and $a_{i}, b_{i j}, c_{i j}$ are constants. The fixed moments of time $m_{k} \in Z$, and satisfy $0<m_{1}<m_{2}<\cdots, \lim _{k \rightarrow \infty} m_{k}=\infty$. The constants $\mu_{i j}(k)$ satisfy the following convergence conditions:

$$
(H): \sum_{k=1}^{\infty} e^{\lambda_{0} k}\left|\mu_{i j}(k)\right|<\infty, \quad i, j=1,2, \ldots,
$$

where $\lambda_{0}$ is a positive constant.

For convenience, we shall rewrite (1) in the vector form:

$$
\left\{\begin{array}{l}
x(m+1)=A x(m)+B f(x(m))+C \sum_{k=1}^{\infty} \mu(k) g(x(m-k)), \quad m \in Z_{\infty}^{+}, \quad m \neq m_{k \prime} \\
x(m+1)=H_{m}(x(m)), \quad m=m_{k \prime} \\
x(m)=\varphi(m), \quad m \in Z_{\infty},
\end{array}\right.
$$

where $x(m)=\left(x_{1}(m), \ldots, x_{n}(m)\right)^{T}, A=\operatorname{diag}\left\{a_{1}, \ldots, a_{n}\right\}, B=\left\{b_{i j}\right\}_{n \times n}, C=\left\{c_{i j}\right\}_{n \times n}, f$ $(x)=\left(f_{1}\left(x_{1}\right), \ldots, f_{n}\left(x_{n}\right)\right)^{T}, g(x)=\left(g_{1}\left(x_{1}\right), \ldots, g_{n}\left(x_{n}\right)\right)^{T}, \mu(k)=\left(\mu_{i j}(k)\right)_{n \times n}, H_{m}(x(m))=$ $\left(H_{1 m}(x(m)), \ldots, H_{n m}(x(m))\right)^{T}, \phi \in C$, and $f(x), g(x), H_{m}(x) \in C\left[R^{n}, R^{n}\right]$.

We will assume that there exists one solution of system (2) which is denoted by $x(m$, $0, \phi)$, or, $x(m)$, if no confusion occurs. We will also assume that $g(0)=0, f(0)=0$ and $H_{m}(0)=0, m=m_{k}$, for the stability purpose of this paper. Then system (2) admits an equilibrium solution $x(m) \equiv 0$.

Definition 2.1. The zero solution of Equation 2 is called globally exponentially stable if there are positive constants $\lambda$ and $M \geq 1$ such that for any initial condition $\phi \in C$, 


$$
\|x(m, 0, \varphi)\| \leq M\|\varphi\| e^{-\lambda m}, \quad m \geq 0 .
$$

Here $\lambda$ is called the exponential convergence rate.

For $A \in R_{+}^{n \times n}$, the spectral radius $\rho(A)$ is an eigenvalue of $A$ and its eigenspace is denoted by

$$
W_{\rho}(A) \triangleq\left\{z \in R^{n} \mid A z=\rho(A) z\right\},
$$

which includes all positive eigenvectors of $A$ provided that the non-negative matrix $A$ has at least one positive eigenvector(see [15]).

Lemma 2.1. [16] Suppose that $M \in R_{+}^{n \times n}$ and $\rho(M)<1$, then there exists a positive vector $z$ such that

$$
(E-M) z>0 .
$$

For $M \in R_{+}^{n \times n}$ and $\rho(M)<1$, we denote

$$
\Omega_{\rho}(M)=\left\{z \in R^{n} \mid(E-M) z>0, z>0\right\},
$$

which is a nonempty set by Lemma 2.1 , and satisfying that $k_{1} z_{1}+k_{2} z_{2} \in \Omega_{\rho}(M)$ for any scalars $k_{1}>0, k_{2}>0$ and vectors $z_{1}, z_{2} \in \Omega_{\rho}(M)$. So $\Omega_{\rho}(M)$ is a cone without vertex in $R^{n}$, we call it a " $\rho$-cone."

Lemma 2.2. Suppose $P \in R_{+}^{n \times n}$ and $Q(k)=\left(q_{i j}(k)\right)_{n \times n}$, where $q_{i j}(k) \geq 0$ and satisfy

$$
\sum_{k=1}^{\infty} e^{\lambda_{1} k} q_{i j}(k)<\infty, \quad i, j=1,2, \ldots, n,
$$

where $\lambda_{1}$ is a positive constant. Denote $Q=\left(q_{i j}\right)_{n \times n} \triangleq\left(\sum_{k=1}^{\infty} q_{i j}(k)\right)_{n \times n} \in R_{+}^{n \times n}$ and let $\rho(P+Q)<1$ and $u(m)=\left(u_{1}(m), \ldots, u_{n}(m)\right)^{T} \in R_{+}^{n}$ be a solution of the following inequality with the initial condition $u\left(m_{0}+m\right) \in C, m \in Z_{\infty}$,

$$
u(m+1) \leq P u(m)+\sum_{k=1}^{\infty} Q(k) u(m-k), \quad m \geq m_{0} .
$$

Then

$$
u(m) \leq z e^{-\lambda\left(m-m_{0}\right)}, \quad m \geq m_{0},
$$

provided that the initial conditions satisfy

$$
u(s) \leq z e^{-\lambda\left(s-m_{0}\right)}, \quad-\infty<s \leq m_{0},
$$

where $z=\left(z_{1}, z_{2}, \ldots, z_{n}\right)^{T} \in \Omega_{\rho}(P+Q), m_{0} \in Z$ and the positive number $\lambda \leq \lambda_{1}$ is determined by the following inequality

$$
\left(e^{\lambda}\left(P+\sum_{k=1}^{\infty} Q(k) e^{\lambda k}\right)-E\right) z<0 .
$$

Proof. Since $\rho(P+Q)<1$ and $P+Q \in R_{+}^{n \times n}$, then, by Lemma 2.1, there exists a positive vector $z \in \Omega_{\rho}(P+Q)$ such that $(E-(P+Q)) z>0$. Using continuity, there must be a sufficiently small constant $\lambda>0$ such that $\left(e^{\lambda}\left(P+\sum_{k=1}^{\infty} Q(k) e^{\lambda k}\right)-E\right) z<0$, i.e., inequality (6) has at least one positive solution $\lambda \leq \lambda_{1}$. 
Let

$$
\gamma(m)=u(m) e^{\lambda\left(m-m_{0}\right)} \quad \text { or } \quad u(m)=\gamma(m) e^{-\lambda\left(m-m_{0}\right)} .
$$

Then, from (5), we have

$$
\gamma(s) \leq z, \quad-\infty<s \leq m_{0} .
$$

By (3), we have

$$
y(m+1)=u(m+1) e^{\lambda\left(m+1-m_{0}\right)} \leq\left(P u(m)+\sum_{k=1}^{\infty} Q(k) u(m-k)\right) e^{\lambda\left(m+1-m_{0}\right)}, \quad m \geq m_{0} .
$$

Since $P+Q \in R_{+}^{n \times n}$, we derive that

$$
\begin{aligned}
y(m+1) & \leq\left(P y(m) e^{-\lambda\left(m-m_{0}\right)}+\sum_{k=1}^{\infty} Q(k) y(m-k) e^{-\lambda\left(m-k-m_{0}\right)}\right) e^{\lambda\left(m+1-m_{0}\right)} \\
& \leq\left(P y(m)+\sum_{k=1}^{\infty} Q(k) y(m-k) e^{\lambda k}\right) e^{\lambda} .
\end{aligned}
$$

We next show for any $m \geq m_{0}$

$$
y(m) \leq z
$$

If this is not true, then there must be a positive constant $m^{*} \geq m_{0}$ and some integer $i$ such that

$$
y_{i}\left(m^{*}+1\right)>z \quad \text { and } \quad \gamma(m) \leq z, \quad-\infty<m \leq m^{*} .
$$

By (6), (9), and the second inequality of (11), we obtain that

$$
\begin{aligned}
y\left(m^{*}+1\right) & \leq\left(\operatorname{P\gamma }\left(m^{*}\right)+\sum_{k=1}^{\infty} Q(k) y\left(m^{*}-k\right) e^{\lambda k}\right) e^{\lambda} \\
& \leq\left(P+\sum_{k=1}^{\infty} Q(k) e^{\lambda k}\right) e^{\lambda} z \leq z,
\end{aligned}
$$

which contradicts the first inequality of (11). Thus (10) holds for all $m \geq m_{0}$. Therefore, we have

$$
u(m) \leq z e^{-\lambda\left(m-m_{0}\right)}, \quad m \geq m_{0},
$$

and the proof is completed.

\section{Main results}

To obtain the global exponential stability of the zero solution of system (2), we introduce the following assumptions.

$\left(A_{1}\right)$ For any $x \in R^{n}$, there exist non-negative diagonal matrices $U$ and $V$ such that

$$
[f(x)]^{+} \leq U[x]^{+}, \quad[g(x)]^{+} \leq V[x]^{+} .
$$

$\left(A_{2}\right)$ For any $x \in R^{n}$, there exist non-negative matrices $R_{k}$ such that

$$
\left[H_{m_{k}}(x)\right]^{+} \leq R_{k}[x]^{+}, \quad k=1,2, \ldots
$$

$\left(A_{3}\right)$ Let $P=[A]^{+}+[B]^{+} U, Q=[C]^{+} \sum_{k=1}^{\infty}[\mu(k)]^{+} V$, and $\rho(P+Q)<1$. 
$\left(A_{4}\right)$ The set $\Omega=\bigcap_{k=1}^{\infty}\left[W_{\rho}\left(R_{k}\right)\right] \bigcap \Omega_{\rho}(P+Q)$ is nonempty.

$\left(A_{5}\right)$ Let

$$
\gamma k \geq \max \left\{1, \rho\left(R_{k}\right)\right\},
$$

and there exists a constant $\gamma$ such that

$$
\frac{\ln \gamma_{k}}{m_{k}-m_{k-1}} \leq \gamma<\lambda, \quad k=1,2, \ldots,
$$

where the positive number $\lambda \leq \lambda_{0}$ is determined by the following inequality

$$
\left(e^{\lambda}\left(P+\sum_{k=1}^{\infty} Q(k) e^{\lambda k}\right)-E\right) z<0, \quad \text { for a given } z \in \Omega .
$$

Theorem 3.1. Assume that the hypothesis $(H)$ and Conditions $\left(A_{1}\right)-\left(A_{5}\right)$ hold. Then the zero solution of (2) is globally exponentially stable and the exponential convergent rate equals $\lambda-\gamma$.

Proof. Since $\rho(P+Q)<1$ and $P+Q \in R_{+}^{n \times n}$, then, by Lemma 2.1, there exists a positive vector $z \in \Omega_{\rho}(P+Q)$ such that $(E-(P+Q)) z>0$. Using continuity and hypothesis $(H)$, there must be a sufficiently small constant $\lambda>0$ such that $\left(e^{\lambda}\left(P+\sum_{k=1}^{\infty} Q(k) e^{\lambda k}\right)-E\right) z<0$, i.e., inequality (14) has at least one positive solution $\lambda$ $\leq \lambda_{0}$.

From (2), Conditions $\left(A_{1}\right)$ and $\left(A_{3}\right)$, we have

$$
\begin{aligned}
{[x(m+1)]^{+} } & \leq[A x(m)]^{+}+[B f(x(m))]^{+}+\left[C \sum_{k=1}^{\infty} \mu(k) g(x(m-k))\right]^{+} \\
& \leq[A]^{+}[x(m)]^{+}+[B]^{+} U[(x(m))]^{+}+[C]^{+} V \sum_{k=1}^{\infty}[\mu(k)]^{+}[x(m-k)]^{+} \\
& =P[x(m)]^{+}+[C]^{+} V \sum_{k=1}^{\infty}[\mu(k)]^{+}[x(m-k)]^{+}, \quad m_{k-1} \leq m \leq m_{k \prime}, \quad k=1,2,3 \ldots
\end{aligned}
$$

where $m_{0}=0$.

For the initial conditions: $x(s)=\phi(s),-\infty<s \leq 0$, where $\phi \in C$, we can get

$$
[x(m)]^{+} \leq d\|\varphi\| e^{-\lambda\left(m-m_{0}\right)}, \quad-\infty<m \leq 0,
$$

where

$$
d=\frac{1}{\min _{1 \leq i \leq n} z_{i}} z_{i} \quad z \in \Omega .
$$

By the property of " $\rho$-cone" and $z \in \Omega \subseteq \Omega_{\rho}(P+Q)$, we have $d\|\phi\| \in \Omega_{\rho}(P+Q)$. Then, all the conditions of Lemma 2.2 are satisfied by (15), (16), and Condition $\left(A_{3}\right)$, we derive that

$$
[x(m)]^{+} \leq d\|\varphi\| e^{-\lambda\left(m-m_{0}\right)}, \quad m_{0} \leq m \leq m_{1} .
$$

Suppose for all $q=1, \ldots, k$, the inequalities

$$
[x(m)]^{+} \leq \gamma_{0} \cdots \gamma_{q-1} d\|\varphi\| e^{-\lambda\left(m-m_{0}\right)}, \quad m_{q-1} \leq m \leq m_{q}
$$


hold, where $\gamma_{0}=1$. Then, from Condition $\left(A_{2}\right)$ and (18), we have

$$
\begin{aligned}
{\left[x\left(m_{q}+1\right)\right]^{+} } & =\left[H_{m_{q}}\left(x\left(m_{q}\right)\right)\right]^{+} \\
& \leq R_{q}\left[x\left(m_{q}\right)\right]^{+} \\
& \leq R_{q} d \gamma_{0} \cdots \gamma_{q-1}\|\varphi\| e^{-\lambda\left(m-m_{0}\right)} .
\end{aligned}
$$

Since $d \in \Omega \subseteq W_{\rho}\left(R_{q}\right)$, we have $R_{q} d=\rho\left(R_{q}\right) d$. Therefore, from (12) and (19), we obtain

$$
\left[x\left(m_{q}+1\right)\right]^{+} \leq \gamma_{0} \cdots \gamma_{q-1} \gamma_{q} d\|\varphi\| e^{-\lambda\left(m-m_{0}\right)} .
$$

This, together with (18), leads to

$$
[x(m)]^{+} \leq \gamma_{0} \cdots \gamma_{k-1} \gamma_{k} d\|\varphi\| e^{-\lambda\left(m-m_{0}\right)}, \quad-\infty<m \leq m_{k}+1 .
$$

By the property of " $\rho$-cone" again, the vector $\gamma_{0} \ldots \gamma_{k-1} \gamma_{k} d \in \Omega_{\rho}(P+Q)$. It follows from (21) and Lemma 2.2 that

$$
[x(m)]^{+} \leq \gamma_{0} \cdots \gamma_{k-1} \gamma_{k} d\|\varphi\| e^{-\lambda\left(m-m_{0}\right)}, \quad m_{k}+1 \leq m \leq m_{k+1} .
$$

yielding, together with (18), that

$$
[x(m)]^{+} \leq \gamma_{0} \cdots \gamma_{k-1} \gamma_{k} d\|\varphi\| e^{-\lambda\left(m-m_{0}\right)}, \quad m_{k} \leq m \leq m_{k+1} .
$$

By mathematical induction, we can conclude that

$$
[x(m)]^{+} \leq \gamma_{0} \cdots \gamma_{k-1} d\|\varphi\| e^{-\lambda\left(m-m_{0}\right)}, \quad m_{k-1} \leq m \leq m_{k}, \quad k=1,2, \ldots
$$

Noticing that $\gamma_{k} \leq e^{\gamma\left(m_{k}-m_{k-1}\right)}$ by (13), we can use (22) to conclude that

$$
\begin{aligned}
{[x(m)]^{+} } & \leq e^{\gamma\left(m_{1}-m_{0}\right)} \cdots e^{\gamma\left(m_{k-1}-m_{k-2}\right)} d\|\varphi\| e^{-\lambda\left(m-m_{0}\right)} \\
& \leq d\|\varphi\| e^{\gamma\left(m-m_{0}\right)} e^{-\lambda\left(m-m_{0}\right)} \\
& =d\|\varphi\| e^{-(\lambda-\gamma)\left(m-m_{0}\right)}, \quad m_{k-1} \leq m \leq m_{k}, \quad k=1,2, \ldots,
\end{aligned}
$$

which implies that the conclusions of the theorem hold.

Remark 3.1. In Theorem 3.1, we may properly choose the matrix $R_{k}$ in the condition $\left(A_{2}\right)$ such that $\Omega \equiv \varnothing$ Especially, when $R_{k}=\alpha_{k} E$ ( $\alpha_{k}$ are non-negative constants), $\Omega$ is certainly nonempty. So, by using Theorem 3.1, we can easily obtain the following corollary.

Remark 3.2. The conditions $\left(A_{1}\right)-\left(A_{5}\right)$ is conservative. For example, we get the absolute value of all coefficients of (2). Recently, the delay-fractioning or delay-partitioning approach $[17,18]$ is widely used that has shown the potential of reducing conservatism. We will combine delay-partitioning approach with difference inequality approach in our future work to reduce the conservatism.

Corollary 3.1. Assume that $(H),\left(A_{1}\right),\left(A_{3}\right)$, and $\left(A_{5}\right)$ hold. For any $x \in R^{n}$, there exist non-negative constants $\alpha_{k}$ such that

$$
\left[H_{m_{k}}(x)\right]^{+} \leq \alpha_{k}[x]^{+}, \quad k=1,2, \ldots
$$

And let $\gamma_{k} \geq\left\{1, \alpha_{k}\right\}$, where the scalar $0<\lambda<\lambda_{0}$ is determined by (14). Then the zero solution of (2) is globally exponentially stable and the exponential convergent rate equals $\lambda-\gamma$.

Proof. Noticing that (23) is a special case of Condition $\left(A_{2}\right)$. Since $\rho\left(R_{k}\right)=\alpha_{k}$, then $W_{\rho}\left(R_{k}\right)=R^{n}$. So, we have $\Omega=\bigcap_{k=1}^{\infty}\left[W_{\rho}\left(R_{k}\right)\right] \bigcap \Omega_{\rho}(P+Q)=\Omega_{\rho}(P+Q)$. Since the 
" $\rho$-cone" $\Omega_{\rho}(P+Q)$ is nonempty by Lemma $2.1,\left(A_{4}\right)$ obviously holds. Thus we can deduce the conclusion in terms of Theorem 3.1.

Remark 3.3. If $H_{k}(x)=x$, then Equation 2 becomes difference equations with distributed delays without impulses in vector form

$$
x(m+1)=A x(m)+B f(x(m))+C \sum_{k=1}^{\infty} \mu_{k} g(x(m-k)),
$$

which contains many popular models such as discrete-time Hopfield neural networks, discrete-time cellular neural networks, and discrete-time recurrent neural networks, and so on.

Corollary 3.2. Assume that $(H),\left(A_{1}\right)$, and $\left(A_{3}\right)$ hold. Then Equation 24 has exactly one equilibrium point, which is globally exponentially stable.

\section{An illustrate example}

In this section, we will give an example to illustrate the global exponential stability of Equation 1 further.

Example. Consider the following difference equation with distributed delays:

$$
\begin{aligned}
x_{1}(m+1)= & \frac{1}{4} x_{1}(m)+\frac{1}{5} \sin \left(x_{1}(m)\right)+\frac{1}{10} x_{2}(m) \\
& -\frac{1}{6} \sum_{k=1}^{\infty} e^{-k}\left|x_{1}(m-k)\right|+\frac{1}{8} \sum_{k=1}^{\infty} e^{-k}\left|x_{2}(m-k)\right|, \quad m \neq m_{k} \\
x_{2}(m+1)= & \frac{1}{5} x_{1}(m)+\frac{1}{6} \sin \left(x_{1}(m)\right)+\frac{1}{8} x_{2}(m) \\
& -\frac{1}{3} \sum_{k=1}^{\infty} e^{-k}\left|x_{1}(m-k)\right|+\frac{1}{10} \sum_{k=1}^{\infty} e^{-k}\left|x_{2}(m-k)\right|
\end{aligned}
$$

with

$$
\begin{aligned}
& x_{1}\left(m_{k}+1\right)=H_{1 m_{k}}\left(x_{1}\left(m_{k}\right), x_{2}\left(m_{k}\right)\right), \\
& x_{2}\left(m_{k}+1\right)=H_{2 m_{k}}\left(x_{1}\left(m_{k}\right), x_{2}\left(m_{k}\right)\right)
\end{aligned}
$$

and $m_{1}=4, m_{k}=m_{k-1}+k$ for $k=2,3, \ldots$. One can check that all the properties given in $(H)$ are satisfied provided that $0<\lambda_{0}<1$.

Case 1. If $H_{i m_{k}}\left(x_{1}, x_{2}\right)=x_{i}$ for $i=1,2$ and $k=1,2, \ldots$, then Equation 25 becomes difference equation with distributed delays without impulses. The parameters of Conditions $\left(A_{1}\right)$ and $\left(A_{3}\right)$ are as follows:

$$
\begin{aligned}
& A=\left(\begin{array}{ll}
\frac{1}{4} & 0 \\
0 & \frac{1}{5}
\end{array}\right), \quad B=\left(\begin{array}{cc}
\frac{1}{5} & \frac{1}{10} \\
\frac{1}{6} & \frac{1}{8}
\end{array}\right), \quad C=\left(\begin{array}{cc}
-\frac{1}{6} & \frac{1}{8} \\
-\frac{1}{3} & \frac{1}{10}
\end{array}\right), \quad U=\left(\begin{array}{ll}
1 & 0 \\
0 & 1
\end{array}\right), \\
& V=\left(\begin{array}{ll}
1 & 0 \\
0 & 1
\end{array}\right), \quad \mu_{k}=\left(\begin{array}{cc}
e^{-k} & 0 \\
0 & e^{-k}
\end{array}\right), \quad P=\left(\frac{9}{20} \frac{1}{10}\right), \\
& Q=\left(\begin{array}{ll}
\frac{1}{6(e-1)} & \frac{1}{8(e-1)} \\
\frac{1}{3(e-1)} & \frac{1}{10(e-1)}
\end{array}\right), \quad P+Q=\left(\begin{array}{ll}
\frac{1}{6(e-1)}+\frac{9}{20} & \frac{1}{8(e-1)}+\frac{1}{10} \\
\frac{1}{3(e-1)}+\frac{1}{6} & \frac{1}{10(e-1)}+\frac{13}{40}
\end{array}\right),
\end{aligned}
$$

where $P=A+[B]^{+} U, Q=[C]^{+} \sum_{k=1}^{m}|\mu(k)| V$. We can easily observe that $\rho(P+Q)=$ $0.8345<1$. By Corollary 3.2, Equation 25 has exactly one globally exponentially stable equilibrium $(0,0)^{T}$. 
Case 2. Next we consider the case where

$$
H_{1 m_{k}}=e^{0.04 k} x_{1}, \quad H_{2 m_{k}}=e^{0.04 k} x_{2} .
$$

We can verify that point $(0,0)^{T}$ is also an equilibrium point of the impulsive difference equation with distributed delays (25)-(26) and the parameters of Conditions $\left(A_{2}\right)$ and $\left(A_{4}\right)$ as follows:

$$
\begin{array}{r}
R_{k}=e^{0.04 k}\left(\begin{array}{ll}
1 & 0 \\
0 & 1
\end{array}\right), \quad \rho\left(R_{k}\right)=e^{0.04 k}, \quad W_{\rho}\left(R_{k}\right)=\left\{\left(z_{1}, z_{2}\right)^{T} \mid z_{1}, z_{2} \in R\right\}, \\
\Omega_{\rho}(P+Q)=\left\{\left(z_{1}, z_{2}\right)^{T}>0 \mid \frac{40(e-1)+40}{81(e-1)-12} z_{1}<z_{2}<\frac{30(e-1)-10}{6(e-1)+30} z_{1}\right\} .
\end{array}
$$

So $\Omega=\left\{\left(z_{1}, z_{2}\right)^{T}>0 \mid z_{2}=z_{1}\right\}$ is not empty. Let $z=(1,1)^{T} \in \Omega$ and $\lambda=0.05$ which satisfies the inequality $\left(\left(e^{\lambda}\left(P+[C]^{+} V \sum_{k=1}^{m}\left|\mu_{k}\right| e^{\lambda k}\right)\right)-E\right) z<0$. We can obtain that for $k=1,2, \ldots$

$$
\gamma_{k}=e^{0.04 k} \geq \max \left\{1, e^{0.04 k}\right\}, \quad \frac{\ln \gamma_{k}}{m_{k}-m_{k-1}} \leq \frac{\ln e^{0.04 k}}{k}=0.04<\lambda .
$$

Clearly, all conditions of Theorem 3.1 are satisfied, so the equilibrium $(0,0)^{T}$ is globally exponentially stable and the exponential convergent rate is equal to 0.01 .

\section{Conclusion}

In this paper, we consider a class of impulsive difference equations with distributed delays. By establishing an impulsive delay difference inequality and using the properties of " $\rho$-cone" and eigenspace of the spectral radius of non-negative matrices, some new sufficient conditions for global exponential stability of the impulsive difference equations with distributed delays are obtained. The conditions $\left(A_{1}\right)-\left(A_{5}\right)$ are conservative. For example, we get the absolute value of all coefficients of (2). We will combine delay-partitioning approach with difference inequality approach in our future work to reduce the conservatism.

\section{Acknowledgements}

The authors would like to thank the referee(s) for his(her) detailed comments and valuable suggestions which considerably improved the presentation of the paper. The study was supported by National Natural Science Foundation of China under Grant 10971147, Scientific Research Fund of Sichuan Provincial Education Department under Grant 10 ZA032 and Fundamental Research Funds for the Central Universities 2010 SCU1006.

\section{Author details}

${ }^{1}$ Yangtze Center of Mathematics, Sichuan University, Chengdu 610064, P. R. China ${ }^{2}$ College of Mathematics and Information Science, Leshan Teachers College, Leshan 614004, P.R. China

\section{Authors' contributions}

Dingshi Li carried out the main proof of the theorems in this paper. Shujun Long carried out the expample. Xiaohu Wang provided the main idea of this paper. All authors read and approve the final manuscript.

\section{Competing interests}

The authors declare that they have no competing interests.

Received: 2 March 2011 Accepted: 17 June 2011 Published: 17 June 2011

\section{References}

1. Kolmanovskii, VB, Shaikhet, LE: Control of systems with aftereffect. In: Translations of Mathematical Monographs, vol. 157,American Mathematical Society, Providence, RI (1996)

2. Xu, DY: Asymptotic behavior of nonlinear difference equations with delays. Comput Math Appl. 42, $393-398$ (2001). doi:10.1016/S0898-1221(01)00164-X 
3. Liz, E, Ferreiro, JB: A note on the global stability of generalized difference equations. Appl Math Lett. 15, 655-659 (2002). doi:10.1016/S0893-9659(02)00024-1

4. Wang, ZD, Liu, YR, Wei, GL, Liu, XH: A note on control of a class of discrete-time stochastic systems with distributed delays and nonlinear disturbances. Automatica. 46, 543-548 (2010). doi:10.1016/j.automatica.2009.11.020

5. Liu, YR, Wang, ZD, Liang, JL, Liu, XH: Synchronization and state estimation for discrete-time complex networks with distributed delays. IEEE Trans Syst Man Cyber B. 38(5):1314-1325 (2008)

6. Wang, Z, Liu, Y, Liu, X: Exponential stabilization of a class of stochastic system with Markovian jump parameters and mode-dependent mixed time-delays. IEEE Trans Automat Control. 55, 1656-1662 (2010)

7. Liu, Y, Wang, Z, Liang, J, Liu, X: Stability and synchronization of discrete-time Markovian jumping neural networks with mixed mode-dependent time-delays. IEEE Trans Neural Netw. 20, 1102-1116 (2009)

8. Zhu, W, Xu, DY, Yang, ZC: Global exponential stability of impulsive delay difference equation. Appl Math Comput. 181, 65-72 (2006). doi:10.1016/j.amc.2006.01.015

9. Zhu, W: Invariant and attracting sets of impulsive delay difference equations with continuous variables. Comput Math Appl. 55, 2732-2739 (2008). doi:10.1016/j.camwa.2007.10.020

10. Song, QK, Cao, JD: Dynamical behaviors of discrete-time fuzzy cellular neural networks with variable delays and impulses. J Franklin Inst. 345, 39-59 (2008). doi:10.1016/j.jfranklin.2007.06.001

11. $\mathrm{Xu}, \mathrm{HL}$, Chen, $\mathrm{YQ}, \mathrm{Teo}, \mathrm{KL}$ : Global exponential stability of impulsive discrete-time neural networks with time-varying delays. Appl Math Comput. 217, 537-544 (2010). doi:10.1016/j.amc.2010.05.087

12. Xu, DY, Zhu, W, Long, SJ: Global exponential stability of impulsive integro-differential equation. Nonlinear Anal. 64, 2805-2816 (2006). doi:10.1016/.na.2005.09.020

13. Zhao, HY: Global asymptotic stability of Hopfield neural network involving distributed delays. Neural Netw. 17, 47-53 (2004). doi:10.1016/50893-6080(03)00077-7

14. Zhang, Q, Wei, XP, Xu, J: Global exponential stability of Hopfield neural networks with continuously distributed delays. Phys Lett A. 315, 431-436 (2003). doi:10.1016/S0375-9601(03)01106-X

15. Horn, RA, Johnson, CR: Matrix Analysis. Cambridge University Press, Cambridge (1985)

16. Lasalle, JP: The Stability of Dynamical System. SIAM, Philadelphia (1976)

17. Wang, Y, Wang, Z, Liang, J: A delay fractioning approach to global synchronization of delayed complex networks with stochastic disturbances. Phys Lett A. 372, 6066-6073 (2008). doi:10.1016/j.physleta.2008.08.008

18. Wang, Z, Wang, Y, Liu, Y: Global synchronization for discrete-time stochastic complex networks with randomly occurred nonlinearities and mixed time-delays. IEEE Trans Neural Netw. 21, 11-25 (2010)

doi:10.1186/1029-242X-2011-8

Cite this article as: Li et al:: Difference inequality for stability of impulsive difference equations with distributed delays. Journal of Inequalities and Applications 2011 2011:8.

\section{Submit your manuscript to a SpringerOpen ${ }^{\circ}$ journal and benefit from:}

- Convenient online submission

- Rigorous peer review

- Immediate publication on acceptance

- Open access: articles freely available online

- High visibility within the field

- Retaining the copyright to your article

Submit your next manuscript at $\boldsymbol{s p r i n g e r o p e n . c o m ~}$ 